\title{
Resonant X-Ray Absorption of Neon Clusters: The Influence of Cluster Size on the Character of Core Excited States
}

\author{
F. Federmann, O. Björneholm, A. Beutler, and T. Möller \\ Hamburger Synchrotronstrahlungslabor HASYLAB am Deutschen, Elektronensynchrotron DESY, Notkestrasse 85, D-22603 \\ Hamburg, Germany
}

(Received 13 May 1994)

\begin{abstract}
First resonant $K$-shell absorption spectra of atomic clusters covering the size range from the atom to the solid are reported. Discrete absorption into Rydberg states as well as the near edge ionization continuum (XANES/EXAFS) of neon clusters $(N \approx 15-4000)$ is investigated with excellent spectral resolution. Shifts and splittings of the absorption features $(1 s \rightarrow 3 p, 4 p)$ provide information on local properties of the different atomic sites. Besides identifying surface and bulk contributions we observe how the character of a transition changes from a Rydberg orbital in a small cluster into a bulk excitation in larger systems.
\end{abstract}

PACS numbers: 78.70.Dm, 36.40.+d, 61.46.+w

In recent years interest in cluster studies as the link between atomic physics and the physics of condensed matter has greatly increased [1]. Of particular interest is the evolution of electronic energy levels with size and their relation to the geometrical structure. In this context rare gas clusters play an important role, as their size can easily be varied from a few atoms to rather large microcrystals [2]. They are model systems for insulators and possess a simple electronic structure without the problems associated with directed bonds or strong electronic correlation as in metals.

Fluorescence spectroscopy of valence shell excitations of rare gas clusters has already provided a wealth of information. Most notably surface and bulk sites have been studied as a function of cluster size [3]. However, despite the simple electronic structure of closed shells, these excitations prove difficult to analyze. The resulting spectra are complicated by spin orbit splitting and the possibility of transitions into $s$ - and $d$-symmetry states. Furthermore, both orbitals involved in a transition are usually modified by clustering, making it difficult to separate the different contributions. This turns out to be impossible for higher excitations which form a broad continuum of overlapping bands [4]. Since clusters - with a large number of nonequivalent atomic sites-also possess a far lower symmetry than the solid, there is an urgent need to simplify the spectra if possible. We will demonstrate in this Letter that many of these problems are avoided when resonant $K$-shell excitations are studied. The resulting absorption spectra are characterized by a single Rydberg series $(1 s \rightarrow n p)$, hence there is no ambiguity concerning the origin of states, and the excitations are localized to a given atom due to the small overlap between core orbitals. However, in order to extract detailed information from the $1 s^{-1} n p$ states these have to be well resolved, requiring highest spectral resolution, which has only recently become available through the development of new monochromators and bright undulator sources for storage rings. Pioneering $K$-shell absorption spectra of molecular clusters show some changes in the region of Rydberg excitations but suffer from low resolution so that no variations due to the cluster size can be observed $[5,6]$.

Neon is the obvious choice for these investigations due to a comparatively low binding energy of $870 \mathrm{eV}$ which results in only moderate lifetime broadening. In the free atom the first two resonances are well resolved. In this paper we study the development of these transitions for a large range of cluster sizes $(\bar{N} \approx 25-3000)$. We observe a distinctly different behavior for these two lowest excitations which can be explained by the respective sizes of the excited orbitals.

The clusters were produced in an adiabatic expansion through a liquid He-cooled conical nozzle (diameter $=100 \mu \mathrm{m}$, opening angle $2 \theta=30^{\circ}$ ) and crossed with monochromated synchrotron radiation after passing through a skimmer into the main vacuum chamber. The average cluster size was varied by choosing appropriate expansion parameters with stagnation pressures ranging from 1 to 3 bars and temperatures down to $37 \mathrm{~K}$. Details of the cluster source have been published elsewhere [7]. The average cluster size in the beam was calculated from the parameters of the expansion using scaling laws [8]. The experiments were performed at the new SX700 undulator beam line BW3 at Hasylab [9]. The resolution used at the neon $K$ edge was typically $300 \mathrm{meV}$, which is comparable to the natural linewidth of the atomic resonance lines of about $250 \mathrm{meV}$ [9]. As a result of this broadening only the $1 s \rightarrow 3 p$ and $1 s \rightarrow 4 p$ transitions are evident in the spectra (cf. Fig. 1). The resolution used is close to the best values reported so far at this energy and in combination with the high flux provides the basis of this investigation.

The yield of cluster ions was recorded with a time of flight ion spectrometer of just $10 \mathrm{~mm}$ length. This compact design allowed us to make direct use of the time structure of synchrotron light without the need 


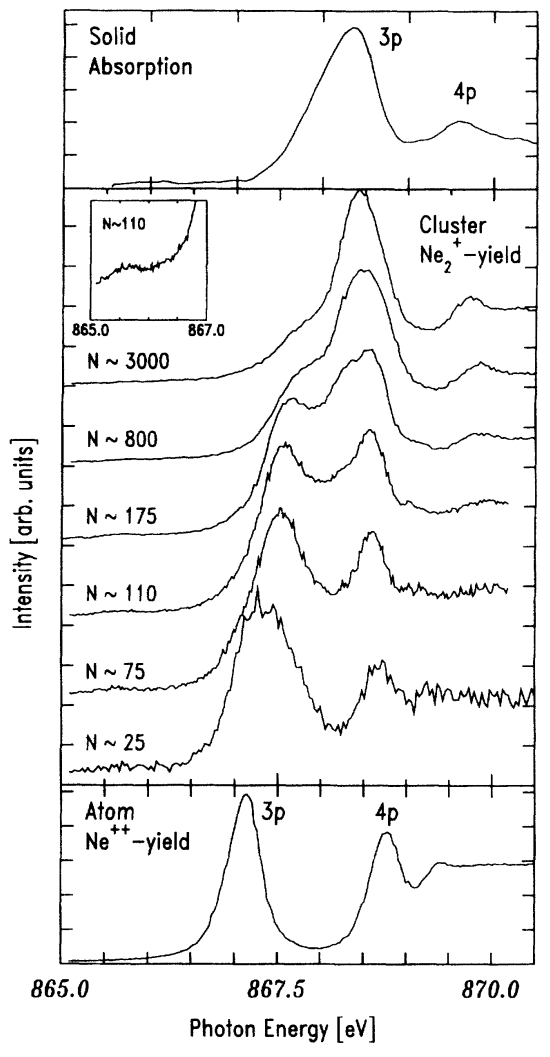

FIG. 1. The development of the resonant $1 s$ excitations in neon from the atom to the solid. The bulk spectrum is taken from Ref. [11]. The inset shows one of the spectra on an expanded scale where a weak feature at around $856.7 \mathrm{eV}$ becomes visible (see text for details). Note that the cluster absorption has been measured on the singly charged neon molecule $\left(\mathrm{Ne}_{2}{ }^{+}\right)$while the yield of the doubly charged atom $\left(\mathrm{Ne}^{++}\right)$is of purely atomic origin.

for electron-ion coincidences. Besides very efficient detection this technique may still be used with very high counting rates - up to 50000 counts/s - without the increased background associated with coincidence experiments. The use of time flight detection also allows the simultaneous recording of spectra of different ions (e.g., $\mathrm{Ne}^{+}, \mathrm{Ne}^{++}, \mathrm{Ne}_{2}{ }^{+}$) since no scanning of the mass spectrometer is required. This is important as an internal reference and allows the accurate determination of shifts relative to the atomic transitions given by the $\mathrm{Ne}^{++}$yield.

It has been shown for the case of argon clusters that weakly bound van der Waals systems fragment strongly after core excitation [10]. The largest part of the signal collects in the dimer ion which therefore reflects the absorption of the entire cluster beam. The same situation is found for neon clusters, and all cluster absorption shown is the actual recorded dimer ion yield. It is not a priori obvious that this method gives correct results under all circumstances. One would for instance expect the real dimer to fragment further to $\mathrm{Ne}^{+}$or $\mathrm{Ne}^{++}$while at the same time a very large cluster could remain stable even when doubly charged. The assignment of an average cluster size to our spectra from the expansion parameters could therefore be inaccurate for very large and the smallest clusters. However, the former should only represent a problem when intensities are compared and we therefore limit ourselves to a discussion of the general features and their energetic position.

The region of resonant $K$-shell absorption in clusters in Fig. 1 and compared to the atom and the bulk solid. The observed resonances are due to transitions from the $1 s$ orbital into unoccupied bound states. For the free atom only a simple, hydrogenlike series, corresponding to the $1 s \rightarrow$ $n p$ transitions, is seen. Compared to the valence shell, where spin orbit splitting is present and transitions into $s$ and $d$-symmetry states are allowed, this is a considerable simplification. This simple character is still preserved for clusters, but the lines are shifted and split into surface and bulk components. A distinction of these two contributions is appropriate due to the fixed position of the core hole which is localized to either of the two sites. For small clusters $(\bar{N}<100)$ little bulk contribution is expected and we observe two resonances that still correspond closely to the atomic spectrum. This interpretation is in analogy to results obtained from valence shell excitations of rare gas clusters where the bands observed in small clusters are assigned to surface transitions [3]. A new feature at around $868.4 \mathrm{eV}$ emerges for intermediate sized clusters $(\bar{N} \approx$ 100) where it overlaps with the original $1 s \rightarrow 4 p$ transition. It becomes more and more prominent with increasing cluster size and is attributed to the $1 s \rightarrow 3 p$ excitation of bulk atoms. In still larger clusters the corresponding $4 p$ transition is observed at around $869.6 \mathrm{eV}$. The spectra of our largest clusters agree well with recent measurements on solid neon [11], showing that the important size range is covered. It should be emphasized that our method of detection is cluster specific and there is no atomic contribution in the spectra.

The results of a fitting procedure are summarized in Fig. 2 where the energetic position of the transitions is shown in dependence of the average cluster size. We note that the $3 p$ bulk excitation seems to be largely unaffected by the overall cluster size, indicating that indeed only the local environment determines the transition. Our estimate for the bulk limit of the $1 s \rightarrow 3 p$ transition of $868.35 \mathrm{eV}$ therefore agrees well with the recently reported result of $868.3 \mathrm{eV}$ by Hiraya et al. [11]. Our experiments also give the first value for the position of the corresponding surface excitation which we place at $867.6 \mathrm{eV}$. A similar relative shift from the free atom to the surface and bulk has been observed for argon, where the surface states are found at about $40 \%$ of the bulk shift [12]. The general trend towards higher energies may be explained by a caging effect of the surrounding atoms which limit the space available to the excited electron. The $1 s \rightarrow 3 p$ surface transition, on the other hand, shows a strong variation 


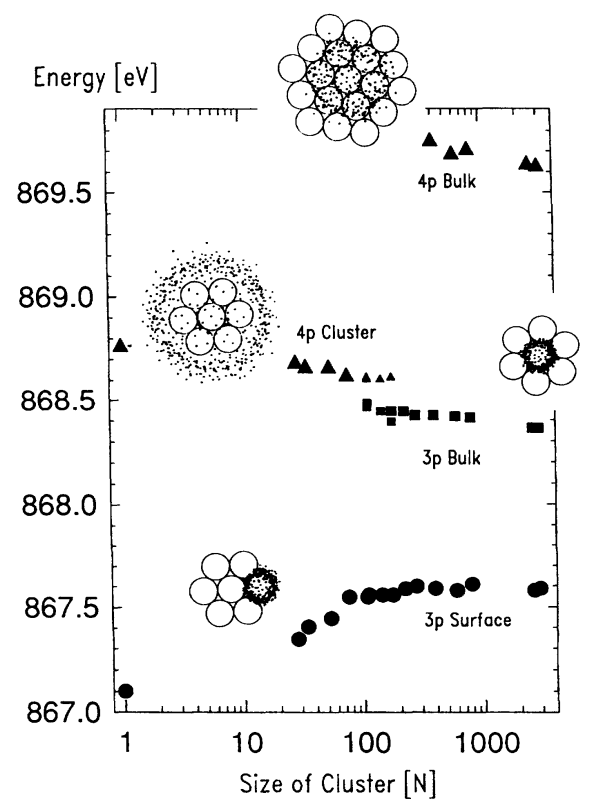

FIG. 2. The shift of the $1 s \rightarrow 3 p, 4 p$ excitations as a function of cluster size. The orbitals and sites of excitation are schematically illustrated. The absolute energy scale was adjusted to agree with EELS data for the atomic $1 s \rightarrow 3 p$ transition [20].

with cluster size. This is not unexpected as the surface of clusters is strongly curved, thus reducing the effective coordination compared to an infinite surface. The effect is particularly efficient for small clusters which is reflected in a correspondingly strong shift. A detailed investigation of the spectra (see inset in Fig. 1) reveals an additional weak feature at $865.7 \mathrm{eV}$ which we associate with the $1 s \rightarrow 3 s$ transition at the surface of the cluster. Dipole forbidden core excitations have also been observed in photoabsorption at the surface of solid argon [13] and argon clusters [12]. The shift of $0.6 \mathrm{eV}$ compared to electron energy loss spectroscopy (EELS) data of the free neon atom [14] is similar to those observed for the other transitions and supports our assignment.

It is interesting to compare the behavior of the $1 s \rightarrow 3 p$ excitation to that of the next member of the series, the $1 s \rightarrow 4 p$ transition. Because the radius of an excited orbital depends strongly on the principal quantum number, this may provide the opportunity to study effects due to the respective sizes of the excited orbital and the cluster as a whole. Indeed, the trend observed is surprising. In contrast to the $3 p$ excitation the $1 s \rightarrow 4 p$ transition shifts downwards in energy. Clearly this cannot at all be explained by caging effects. When calculating approximate sizes of the atomic orbitals, using the quantum defects for sodium $(Z+1$ approximation [15]), we arrive at a value of $9.5 \AA$ for the expectation value in the $\left(1 s^{-1}\right) 4 p$ state. This compares with a nearest neighbor distance of $3.1 \AA$ in the solid and shows that-even though the wave functions are likely modified in the cluster-the orbital may well extend beyond the second coordination sphere $(N=55)$. For very small clusters the $4 p$ state will thus largely be located outside the cluster, acting as a Rydberg orbital to the whole system. The energy required for the corresponding excitation will be reduced due to the screening of the core hole by the other atoms in agreement with our results. As the cluster grows this Rydberg orbital no longer exists and the $4 p$ excitation will form a new state inside the cluster at correspondingly higher energy. This interpretation agrees well with our data showing a drop in the $4 p$ intensity at a few hundred atoms and the simultaneous emergence of a new structure at higher energies $(869.6 \mathrm{eV})$.

So far in the discussion we assumed the clusters to be solid. While this has been shown for argon clusters produced in an adiabatic expansion [16], the situation is not so clear for neon due to the lower mass and increased zero point vibrational energy. Recent investigations in our group hint to a phase change from liquid to solid for Xedoped neon clusters [17]. Structure related information can be extracted from the intensity variations of the $1 s$ ionization continuum in the near edge region which is known as $\mathrm{x}$-ray appearance near edge (XANES) or extended $\mathrm{x}$-ray absorption fine structure spectroscopy (EXAFS) (Fig. 3). This has been demonstrated in principle for argon clusters, where the unselected ion yield of relatively large clusters has been used in an EXAFS analysis [18]. In our spectra only slight undulations are observed for small clusters while more structure continuously emerges for larger systems, where the agreement with data collected on solid

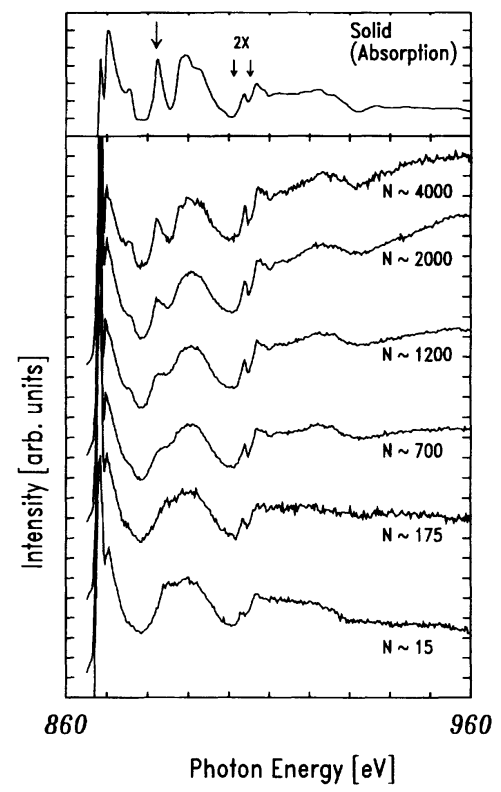

FIG. 3. Extended x-ray absorption spectra for clusters of various sizes and the solid [11]. The first arrow marks the peak discussed in the text, the others the approximate position of double excitations in the free atom. 
neon films is very good [11]. The reduced amplitude of the oscillations is expected due to the large proportion of surface atoms even in "large" clusters. The two features seen around $905 \mathrm{eV}$ are probably related to atomic double excitations.

In a recent paper by Soldatov et al. [19] the XANES structure of solid neon and single atoms surrounded by a given number of further shells is calculated. The computations include multiple scattering and double excitations. The results indicate that the peak at $882 \mathrm{eV}$ is caused by atoms in the second coordination sphere of the fcc lattice and would not be observed if only nearest neighbors were present. The fact that our largest clusters show practically the same spectra therefore reflects a high degree of internal order beyond the first coordination sphere and rules out the possibility of liquid droplets for the larger systems $(N>1000)$. Whether it is also possible by this means to study the expected transition from icosahedral to fcc structure will be the subject of future investigations.

In conclusion, we have shown that high resolution $K$-shell absorption spectra of neon clusters provide valuable information on the electronic and geometric structure. The simple character of the underlying transitions allows the study of cluster properties from the free atom to the solid. We were thus able to show how a transition changes its character from a Rydberg type excitation of the whole cluster to a bulk excitation of the solid. In addition, extrapolation of the data provides first spectroscopic information on core excited surface states of solid neon. The high flux and excellent resolution of modern monochromators and undulators, now becoming available will undoubtedly stimulate further research into $K$-shell spectroscopy of atomic and molecular clusters.

[1] Clusters of Atoms and Molecules I, edited by H. Haberland, Springer Series in Chemical Physics Vol. 52 (Springer, Berlin, 1994).
[2] J. Jortner, Z. Phys. D 24, 247 (1992).

[3] J. Stapelfeldt, J Wörmer, and T. Möller, Phys. Rev. Lett. 62, 98 (1989).

[4] J. Wörmer, M. Joppien, G. Zimmerer, and T. Möller, Phys. Rev. Lett. 67, 2053 (1991).

[5] E. Rühl, Ber. Bunsenges. Phys. Chem. 96, 1172 (1992).

[6] E. Rühl, C. Schmale, H. W. Jochims, E. Biller, R. Locht, A.P. Hitchcock, and H. Baumgärtel, in Synchrotron Radiation and Dynamic Phenomena, AIP Conference Proceedings No. 258 (AIP, New York, 1992).

[7] R. Karnbach, M. Joppien, J. Stapefeldt, J. Wörmer, and T. Möller, Rev. Sci. Instrum. 64, 2838 (1993).

[8] O.F. Hagena, Z. Phys. D 4, 291 (1987). The average cluster size is obtained with the additional empiric relation $\bar{N}=38\left(\Gamma^{*} / 1000\right)^{2}$.

[9] C. U.S. Larsson, A. Beutler, O. Björneholm, F. Federmann, U. Hahn, A. Rieck, S. Verbin, and T. Möller, Nucl. Instrum. Methods Phys. Res., Sect. A 337, 603 (1994).

[10] E. Rühl, C. Heinzel, A. P. Hitchcock, and H. Baumgärtel, J. Chem. Phys. 98, 2653 (1993).

[11] A. Hiraya, K. Fukui, P. K. Tseng, T. Murata, and M. Watanabe, J. Phys. Soc. Jpn. 60, 1824 (1991).

[12] O. Björneholm, F. Federmann, F. Fössing, and T. Möller (to be published).

[13] W. Wurth, G. Rocker, P. Feulner, R. Scheuerer, I. Zhu, and D. Menzel, Phys. Rev. B 47, 6697 (1993).

[14] A. P. Hitchcock and C. E. Brion, J. Phys. B 13, 3269 (1980).

[15] W.H.E. Schwarz, Angew. Chemie Int. Ed. 13, 454 (1974).

[16] J. Farges, M. F. de Feraudy, B. Raoult, and G. Torchet, J. Chem. Phys. 84, 3491 (1986).

[17] R. von Pietrowski, Diplomarbeit Universität Hamburg, 1993.

[18] E. Rühl, C. Heinzel, A.P. Hitchcock, H. Schmelz, C. Reynaud, H. Baumgärtal, W. Drube, and R. Frahm, J. Chem. Phys. 98, 6820 (1993).

[19] A. V. Soldatov, T. S. Ivanchenko, S. Della Longa, and A. Bianconi, Phys. Rev. B 47, 16155 (1993).

[20] R. N. S. Sodhi and C. E. Brion, J. Electron. Spectrosc. 34, 363 (1984). 


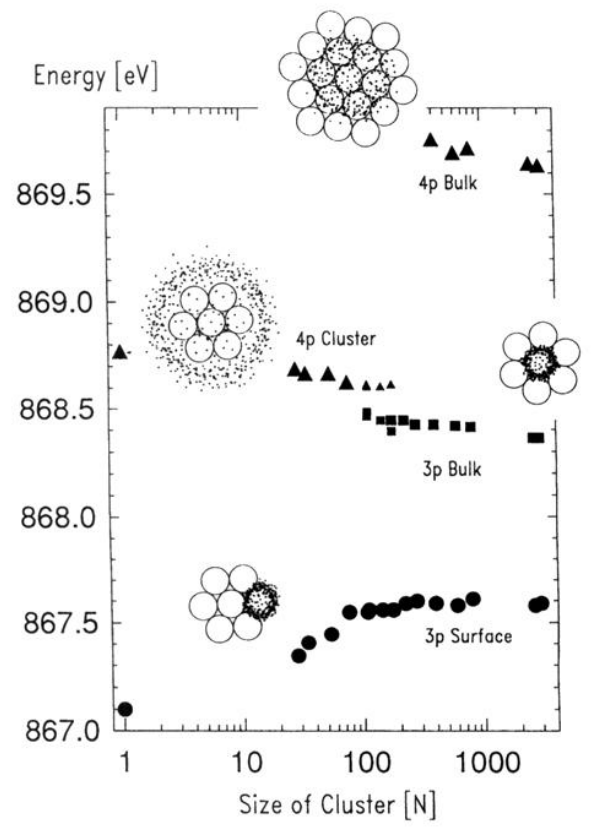

FIG. 2. The shift of the $1 s \rightarrow 3 p, 4 p$ excitations as a function of cluster size. The orbitals and sites of excitation are schematically illustrated. The absolute energy scale was adjusted to agree with EELS data for the atomic $1 s \rightarrow 3 p$ transition [20]. 